\title{
Cyclic Performance Study of Silicon/Carbon Nanotube Composite Anodes Using Electrochemical Impedance Spectroscopy
}

\author{
U. Tocoglu*, O. Cenher, T. Cetinkaya, M.O. Guler and H. Akbulut \\ Sakarya University, Engineering Faculty, Department of Metallurgical and Materials Engineering \\ Esentepe Campus 54187, Turkey
}

\begin{abstract}
Silicon based carbon nanotube composites were produced and their galvanostatic cycling properties analyzed depended on the electrochemical impedance spectroscopy. Composite anodes were produced via vacuum filtration and DC magnetron sputtering technique. Carbon nanotube papers were produced with vacuum filtration as substrate for silicon deposition and silicon was sputtered onto carbon nanotube papers via DC magnetron sputtering. Scanning electron microscopy and X-ray diffraction analysis were conducted for structural analysis of anodes. CR2016 coin cells were assembled for electrochemical tests. Electrochemical performance of anodes was tested via galvanostatic charge/discharge (100 cycles) analysis. Electrochemical impedance spectroscopy was carried out at every 25 charge/discharge cycle to determine relation between cyclic performance and electrochemical impedance of cells.
\end{abstract}

DOI: 10.12693 /APhysPolA.125.290

PACS: 82.47.Aa

\section{Introduction}

Today's most widely used powering tool of electronic devices is lithium ion batteries. Due to their advantages like high energy density and showing no memory effect, lithium ion batteries are the first choice of both consumers and producers. Despite of outstanding properties of those electrochemical energy storage devices, they are still needed to be developed in terms of energy storage capability and life span. Currently the most important concern about the environmental issues is $\mathrm{CO}$ and $\mathrm{CO}_{2}$ emission of motor vehicles. Also the fossil fuel sources are exhausting rapidly and the human being has already started to search for new energy sources and more efficient energy storage devices [1].

Because of the problems about energy and environment, electrical vehicles have attracted more attention and lithium ion batteries are the best energy storage candidate for them. Although lithium ion batteries already meet the energy requirements of portable devices sufficiently, they still need to come a long way to meet the requirements of electrical vehicles. Many researchers are trying to improve performance of lithium ion batteries by means of developing novel cathode, anode and electrolyte materials. In case of commercial lithium ion battery graphite anode, it exhibits excellent capacity retention during cycling of battery but it could not deliver sufficient energy density for modern electrical vehicle applications due to its low theoretical capacity $(372 \mathrm{mAh} / \mathrm{g})$.

Many anode materials are being investigated to replace graphite anode to meet the energy storage requirements

*corresponding author; e-mail: utocoglu@sakarya.edu.tr of lithium ion batteries. Silicon is the one of the most important candidate of next generation lithium ion battery anodes with its highest known theoretical capacity of $4200 \mathrm{mAh} / \mathrm{g}$ [2]. Despite of nearly ten times higher capacity value than graphite, there is a great obstacle which hinders silicon to become practical battery anode and commercialization. During lithiation of silicon anode, volume variations could reach $300 \%$ of its original state [3]. Volume variations caused, because of the lithiation leads, the mechanical stresses and causes cracking and pulverization of the anode. Losing the mechanical integrity because of the cracking results with electrical contact loss and rapid capacity fade.

Novel anode architectures have been suggested in order to solve the volume variation related problems of silicon anodes. Thin film and nanosized silicon anodes have been studied extensively but it is understood that they could not provide enough mechanical integrity during lithiation [4].

The superior mechanical properties of carbon nanotubes (CNTs) have received much attention, leading to many efforts to design materials that realize macroscale advantages through integrating nanoscale composite structures. Core/shell (Si/CNT) composite architecture has many advantages against the volume variations of silicon. The mechanical stresses could be absorbed by carbon nanotubes while the conductive carbon nanotube network provides the superior electrical conductivity.

In this study it is aimed to produce carbon nanotube reinforced silicon anodes to enhance the electrochemical performance of silicon anodes. It is also aimed to achieve superior cycle life compared that of conventional silicon anodes with the optimized nanoscale composite structures [5]. 


\section{Experimental}

Carbon nanotube papers (buckypaper) were produced from multiwalled carbon nanotubes (MWCNT) as substrate of sputtering process. Buckypaper production consists of three steps as follows: purification, functionalization, and vacuum filtration. After buckypaper production step, DC magnetron sputtering technique was employed for composite electrode production. Silicon was sputtered onto buckypapers from silicon targets. Two different DC powers were chosen for sputtering process as $100 \mathrm{~W}$ and $200 \mathrm{~W}$ with constant deposition time. Phase analysis and crystal structure investigations of composite anodes were carried out via XRD (Rigaku D/MAX 2000). Morphology analyses of composite anode structures were carried out via scanning electron microscopy (SEM) (JEOL JSM-6060 LV system). Coin type (CR2016) cells were assembled for electrochemical characterization of composite anodes. Composites anodes were used as working electrode and pure lithium foils were used as counter electrode. $1 \mathrm{M} \mathrm{LiPF}_{6}$ dissolved in a mixture of ethylene carbonate (EC) and diethyl carbonate (DEC) (1:1 by wt) solution was chosen as the electrolyte. Galvanostatic charge/discharge tests were carried out by MTI Model BST8-MA electrochemical analyzer with constant current density between voltage ranges of $0.05-2.0 \mathrm{~V}$. Cyclic voltammetry analyses were carried out with Gamry Instruments Reference 3000 electrochemical work station between voltage ranges of 0.05-2.0 V. Electrochemical impedance studies were carried out to associate the capacity retention with cell impedance.

\section{Results and discussion}

XRD pattern of $\mathrm{Si} / \mathrm{MWCNT}$ nanocomposite anodes is shown in Fig. 1. The pattern shows only carbon nanotube reflections (002) which means that the deposited silicon layer on carbon nanotubes is amorphous. Amorphous structure is more beneficial than crystalline structure for electrochemical performance of anode since it has more open structure to allow lithium ions diffuse through the electrode during the cycling of battery [6]. So that provides the more efficient reactions to take place between electrolyte and anode which provides higher energy densities.

SEM images of composite anodes produced with different sputtering powers are presented in Fig. 2. It could be understood from the images that composite anodes have high degree porous structure. Carbon nanotube branches of buckypapers were wrapped around by a silicon layer. Also we can conclude that thickness of silicon layer has increased with the increase of sputtering power. Such high degree porous electrode structure provides electrochemical superiorities in comparison with the nonporous structures. The volume increment of silicon phase of anode could be hosted at pores and mechanical failure of anode could be prevented. Also porous structure pro-

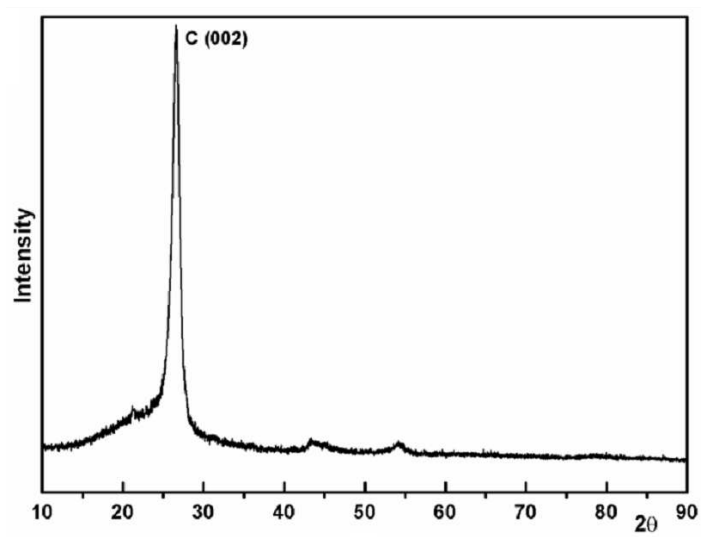

Fig. 1. XRD pattern of $\mathrm{Si} / \mathrm{MWCNT}$ composite electrodes.

vides greater surface area so the more efficient interaction could take place between electrodes and electrolyte [7].

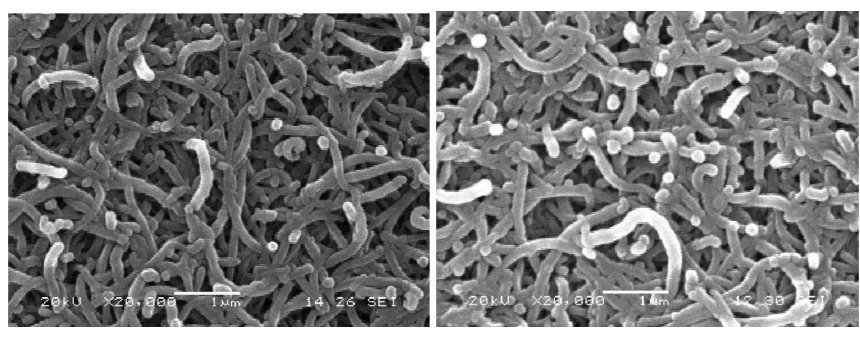

Fig. 2. SEM images of composite anodes produced with different sputtering power (a) $100 \mathrm{~W}$, (b) $200 \mathrm{~W}$.

Figure 3 shows the galvanostatic charge/discharge behaviors of composite anodes produced via magnetron sputtering technique. Anodes both produced with $100 \mathrm{~W}$ and $200 \mathrm{~W}$ sputtering powers have shown more capacity values than commercial graphite anodes. Even after 100 galvanostatic charge/discharge cycles anodes have shown significant capacity values. Anode which was produced with $100 \mathrm{~W}$ sputtering power has exhibited $2482 \mathrm{mAh} \mathrm{g}^{-1}$ specific capacity after first discharge. After second cycle discharge value exhibited a huge drop due to the formation of solid electrolyte phase (SEI) formation on the anode surface.

Formation of SEI causes the consumption of electrolyte and decreases reversible capacity of cell. Anode which was produced with $100 \mathrm{~W}$ DC power showed $805 \mathrm{mAh} \mathrm{g}^{-1}$ capacity at second cycle and showed $606 \mathrm{mAh} \mathrm{g}^{-1}$ capacity value after 100 cycles. Regarding the anode which was produced with $200 \mathrm{~W}$ DC power it has exhibited capacity values 2439,820 , and $452 \mathrm{mAh} \mathrm{g}^{-1}$, respectively, after first, second, and last cycle. It could be concluded from comparison of capacity values between two electrodes, lower sputtering power yielded better cycle performance. The reason for better performance of anode which was produced with lower sputtering power is lower silicon layer thickness around 


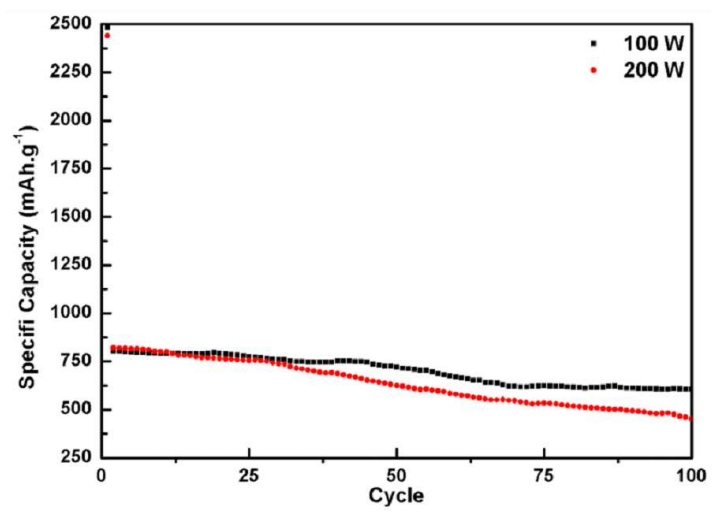

Fig. 3. Cyclic performance plots of anodes which were produced with $100 \mathrm{~W}$ and $200 \mathrm{~W}$ sputtering powers.

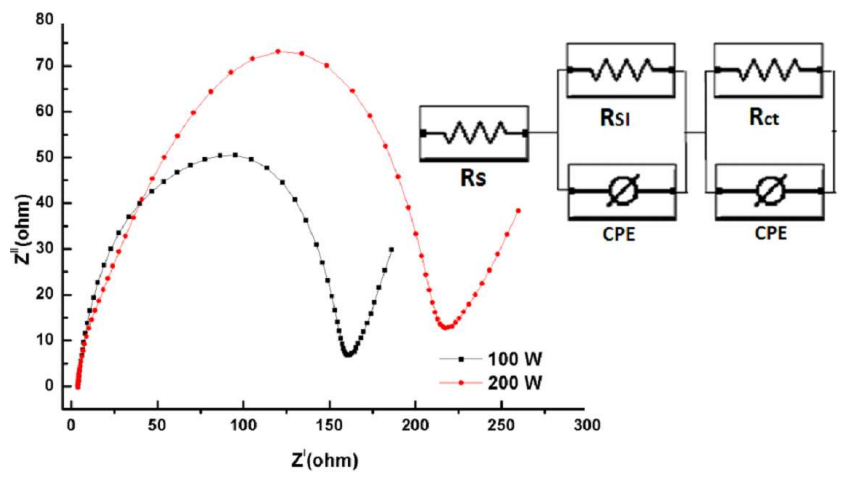

Fig. 4. Nyquist plots of cells and the equivalent circuit of impedance spectra.

the carbon nanotubes. This result is similar to the thin film anode studies at literature and our previous studies. Thinner active material layer is more durable to the volume variation induced mechanical stresses [8].

Figure 4 represents the Nyquist plots of electrochemical impedance spectroscopy results and the fitted circuit to the impedance spectra. The impedance circuit consists of resistance elements such as $R_{\mathrm{s}}$ which corresponds to total ohmic resistance of cell components, $R_{\mathrm{si}}$ which corresponds to resistance of passive surface film on electrodes, and $R_{\text {ct }}$ which corresponds to charge transfer resistance at the electrodes. The $R_{\mathrm{S}}$ value is practically the same for two cells since the components are same. $R_{\mathrm{si}}$ was increased with the increasing sputtering power because the thicker silicon layer caused the denser passive surface film on the anode. Finally, $R_{\mathrm{ct}}$ value was increased with the increase of sputtering power. The thicker silicon layer has reduced porous area of the electrodes so the charge transfer of cell has increased. The impedance test result fits well with the galvanostatic charge/discharge results. The anode which has the higher impedance values has showed the worse electrochemical cycling performance.

\section{Conclusions}

Composite silicon/carbon nanotube anodes were successfully produced via magnetron sputtering technique. XRD analysis has showed that the silicon layer coated around the carbon nanotubes was amorphous which is beneficial for cycling performance of anode. The galvanostatic charge/discharge tests showed that the lower sputtering power has led the better cycling performance due to the higher resistance of thinner active material layer around the carbon nanotubes. Electrochemical impedance results verified the thickness effect on the cell performance.

\section{Acknowledgments}

This work is supported by the Scientific and Technological Research Council of Turkey (TÜBİTAK) under the contract number $111 \mathrm{M} 021$. The authors thank the TÜBİTAK MAG workers for their financial support.

\section{References}

[1] C. Kang, I. Lahiri, R. Baskaran, W.G. Kim, Y.K. Sun, W. Choi, J. Power Sources 219, 364 (2012).

[2] H. Wu, Y. Cui, Nano Today 7, 414 (2012).

[3] S.H. Nguyen, J.C. Lim, J.K. Lee, Electrochim. Acta 74, 53 (2012).

[4] Z. Du, S. Zhang, T. Jiang, R. Lin, J. Zhao, Electrochim. Acta 74, 222 (2012).

[5] R. Epur, M.K. Datta, P.N. Kumta, Electrochim. Acta 85, 680 (2012).

[6] V. Baranchugov, E. Markevich, E. Pollak, G. Salitra, D. Aurbach, Electrochem. Commun. 9, 796 (2007).

[7] M. Thakura, M. Isaacson, S.L. Sinsabaugh, M.S. Wonga, S.L. Biswala, J. Power Sources 205, 426 (2012).

[8] M.S. Park, G.X. Wang, H.K. Liu, S.X. Doua, Electrochim. Acta 51, 5246 (2006). 